\title{
INERTIAL COEFFICIENT RINGS AND THE IDEMPOTENT LIFTING PROPERTY ${ }^{1}$
}

\author{
ELLEN E. KIRKMAN
}

\begin{abstract}
A commutative ring $R$ with identity is called an inertial coefficient ring if every finitely generated $R$-algebra $A$ with $A / N$ separable over $R$ contains a separable $R$-subalgebra $S$ of $A$ such that $A=S+N$, where $N$ is the Jacobson radical of $A$. We say $A$ has the idempotent lifting property if every idempotent in $A / N$ is the image of an idempotent in $A$. Our main theorem is that any finitely generated algebra over an inertial coefficient ring has the idempotent lifting property.
\end{abstract}

All rings contain an identity; all subrings contain the identity of the overring; all homomorphisms preserve the identity. Throughout $R$ denotes a commutative ring and $A$ an $R$-algebra which is finitely generated as an $R$-module. The Jacobson radical of a $\operatorname{ring} B$ is $\operatorname{denoted} \operatorname{rad}(B)$ and throughout $\operatorname{rad}(A)=N$. A separable $R$-subalgebra $S$ of $A$ such that $A=S$ $+N$ is called an inertial subalgebra. If every finitely generated $R$-algebra $A$ with $A / N R$-separable has an inertial subalgebra, $R$ is called an inertial coefficient ring. The basic properties of inertial subalgebras and inertial coefficient rings can be found in [7].

If $I$ is an ideal of a ring $B$ we call $(B, I)$ an $L$. $I$. pair (lifting idempotent pair) if every idempotent in the factor ring $B / I$ is the image of an idempotent in $B$; if $(A, N)$ is an L. I. pair we say $A$ has the idempotent lifting property.

Our main theorem is motivated by a conjecture of E. C. Ingraham that if every finitely generated $R$-algebra has the idempotent lifting property then $R$ is an inertial coefficient ring. Our proof of the converse of this conjecture has as a corollary that an inertial coefficient ring is a Hensel ring (see [5], [6], and [10] for definition and properties of Hensel rings), recalling the role Hensel local rings have played in generalizations of the Wedderburn Principal Theorem by Azumaya, Ingraham, and W. C. Brown. A second immediate consequence of our main theorem is that when $R$ is an inertial coefficient ring, two inertial subalgebras of an $R$-algebra $A$ are conjugate under an inner automorphism of $A$, generalizing Malcev's uniqueness statement to Wedderburn's Principal Theorem.

Received by the editors April 14, 1976.

AMS (MOS) subject classifications (1970). Primary 16A16, 16A32; Secondary 13J15.

Key words and phrases. Inertial coefficient ring, lifting idempotents, Hensel ring, separable algebra.

${ }^{1}$ This material appeared in my doctoral dissertation written at Michigan State University. I am deeply indebted to Professor E. C. Ingraham for his guidance in its preparation. 
Our main theorem is proved in three steps. We first consider the case where $R$ is an inertial coefficient ring which contains no idempotents but 0 and 1 , (i.e. $R$ is a connected ring) and show $R / \operatorname{rad}(R)$ is connected. We next use the Pierce representation of $R$ as the ring of global sections of a sheaf of connected rings [12] to show that if $R$ is any inertial coefficient ring, an idempotent in $R / \operatorname{rad}(R)$ is the image of an idempotent in $R$. Finally we show that any finitely generated $R$-algebra has the idempotent lifting property.

THEOREM 1. Let $R$ be a connected ring. If $R / \operatorname{rad}(R)$ has an idempotent not equal to 0 or 1 then $R$ is not an inertial coefficient ring.

Proof. By [9, Proposition 4, p. 54] there exists a $p \in \operatorname{rad}(R)$ such that $x^{2}-x+p \in R[x]$ has no root in $\operatorname{rad}(R)$. Let $f(x)=x^{3}-x^{2}+p x$. Reducing the coefficients of $f(x)$ modulo $\operatorname{rad}(R)$ we obtain, $\bar{f}(x)=x^{2}(x-1)$, but it is easy to check that there do not exist monic polynomials $g(x)$ and $h(x)$ contained in $R[x]$ such that $f(x)=g(x) h(x)$, with $\bar{g}(x)=x^{2}$ and $\bar{h}(x)=$ $x-1$.

Let $A$ be the finitely generated, faithful, free, commutative $R$-algebra $A=R[x] /\langle f(x)\rangle$. Using the Chinese Remainder Theorem we obtain

$$
A /(\operatorname{rad}(R) A) \cong\left((R / \operatorname{rad} R)[x] /\left\langle x^{2}\right\rangle\right) \oplus((R / \operatorname{rad} R)[x] /\langle x-1\rangle) .
$$

Since $\operatorname{rad}(R) \cdot A \subseteq N, A / N \cong R / \operatorname{rad}(R) \oplus R / \operatorname{rad}(R)$, and hence $A / N$ is $R$-separable.

We shall show that the assumption that $A$ has an inertial subalgebra $S$ leads to a contradiction. By [7, Proposition 2.8, p. 80] if such an $S$ exists, it must be a projective $R$-module and by [2, proof of Lemma 1, p. 11] $\operatorname{rank}_{R} S=2$, where by $\operatorname{rank}_{R} S$ we mean the dimension of the free $R_{m}$-module $S_{m}$, for any maximal ideal $m$ of $R$ [3, Theorem 4.12, p. 32].

Case 1. Assume $S$ is a connected ring. By the "projective lifting property" of separable algebras [3, Proposition 2.3, p. 48], $A$ is a projective $S$-module. Since $S$ is connected, $\operatorname{rank}_{S}(A)$ is defined, and the multiplicative property of rank [3, Exercise 2, p. 35] yields $3=\operatorname{rank}_{R}(A)=\operatorname{rank}_{R}(S) \operatorname{rank}_{S}(A)=$ $2 \operatorname{rank}_{S}(A)$. Thus 2 divides 3 , a contradiction.

Case 2. Assume $S$ has an idempotent $e \neq 0,1$. Since $\operatorname{rank}_{R}(S e)=1$ and since $R e$ is an $R e$-direct summand of $S e$ [3, Corollary 4.2, p. 56], we have $S e \cong R e$ as rings. Similarly $S(1-e) \cong R(1-e)$, so $S \cong R e \oplus R(1-e)$ (as rings). We now show that we can assume $e$ to be of the form $a_{1} x+a_{2} x^{2}$ where $a_{1} \in \operatorname{rad}(R)$ and $a_{2}$ is a unit of $R$ such that $\bar{a}_{2}$ is an idempotent in $R / \operatorname{rad}(R)$.

First, writing $e=a_{0}+a_{1} x+a_{2} x^{2}$ with $a_{i} \in R$, we have

$$
\begin{aligned}
0= & e^{2}-e=\left(a_{0}^{2}-a_{0}\right)+\left(2 a_{0} a_{1}-a_{1}\right) x \\
& +\left(a_{1}^{2}-a_{2}+2 a_{0} a_{2}\right) x^{2}+\left(2 a_{1} a_{2}\right) x^{3}+\left(a_{2}^{2}\right) x^{4} .
\end{aligned}
$$

Now applying the relations $x^{3}=x^{2}-p x$ and $x^{4}=(1-p) x^{2}-p x$ we obtain 


$$
\begin{aligned}
0= & \left(a_{0}^{2}-a_{0}\right)+\left(2 a_{0} a_{1}-a_{1}-2 a_{1} a_{2} p-a_{2}^{2} p\right) x \\
& +\left(a_{1}^{2}-a_{2}+2 a_{0} a_{1}+2 a_{1} a_{2}+a_{2}^{2}(1-p)\right) x^{2} .
\end{aligned}
$$

This equation yields the following relations:

$$
\begin{gathered}
a_{0}^{2}=a_{0}, \\
2 a_{0} a_{1}-a_{1}-2 a_{1} a_{2} p-a_{0}^{2} p=0, \\
a_{1}^{2}-a_{2}+2 a_{0} a_{2}+2 a_{1} a_{2}+a_{2}^{2}(1-p)=0 .
\end{gathered}
$$

Since $R$ is connected, equation (1) implies $a_{0}=0$ or $a_{0}=1$ and equations (2) and (3) imply $e$ or $1-e$ is of the form $a_{1} x+a_{2} x^{2}$ where $a_{1} \in \operatorname{rad}(R)$ and $\bar{a}_{2}$ is an idempotent in $R / \operatorname{rad}(R)$.

Second, one can check that $A / N \cong(R / \operatorname{rad} R)[x] /\left\langle x^{2}-x\right\rangle$. From $A=S$ $+N$ it follows that there exist $r_{1}, r_{2} \in R$ and $n \in N$ such that $x=r_{2}+$ $\left(r_{1}-r_{2}\right) e+n$. Since $\bar{e}=\bar{a}_{2} \bar{x}^{2}$ in $A /(\operatorname{rad}(R) A)$, we have

$$
\bar{x}=\bar{r}_{2}+\left(\bar{r}_{1}-\bar{r}_{2}\right) \bar{a}_{2} \bar{x}^{2}+\bar{n} \quad \text { in } A /(\operatorname{rad}(R) A)
$$

and

$$
\overline{\bar{x}}=\overline{\bar{r}}_{2}+\left(\overline{\bar{r}}_{1}-\overline{\bar{r}}_{2}\right) \overline{\bar{a}}_{2} \overline{\bar{x}} \text { in } A / N,
$$

where $\overline{\bar{a}}$ denotes the image of $a \in A$ in $A / N$. Hence $\overline{\bar{r}}_{2}=\overline{\overline{0}}$ and $\overline{\overline{1}}=\overline{\bar{r}}_{1} \overline{\bar{a}}_{2}$. Since $\overline{\bar{a}}_{2}$ is both a unit and an idempotent of $A / N, \overline{\bar{a}}_{2}=\overline{\overline{1}}$. But then by $[5$, Lemma 1.2, p. 46], $\bar{a}_{2}=\overline{1}$ in $A /(\operatorname{rad}(R) A)$ and, hence, $\bar{e}=\bar{x}^{2}$ and $a_{2}$ is a unit of $R$.

Thus

$$
\begin{aligned}
A & =A e \oplus A(1-e)=\left\langle a_{2} x^{2}+a_{1} x\right\rangle \oplus\left\langle a_{2} x^{2}+a_{1} x-1\right\rangle \\
& =\left\langle x^{2}+a_{2}^{-1} a_{1} x\right\rangle \oplus\left\langle x^{2}+a_{2}^{-1} a_{1} x-a_{2}^{-1}\right\rangle .
\end{aligned}
$$

Furthermore

$$
x^{2}+a_{2}^{-1} a_{1} x \equiv x^{2} \quad \operatorname{modulo} \operatorname{rad}(R) A
$$

and

$$
x^{2}+a_{2}^{-1} a_{1} x-a_{2}^{-1} \equiv x^{2}-1 \quad \operatorname{modulo} \operatorname{rad}(R) A .
$$

Finally $\left\langle x^{2}-1\right\rangle=\langle x-1\rangle$ in $A / \operatorname{rad}(R) A$, since $\left\langle x^{2}-1\right\rangle \subseteq\langle x-1\rangle$ and $(1-x)\left(x^{2}-1\right)=(x-1)$ in $A /(\operatorname{rad}(R) A)$ implies $\langle x-1\rangle \subseteq\left\langle x^{2}-1\right\rangle$. Thus by [5, proof of Theorem 3.1, p. 54] there exist monic polynomials $g(x)$ and $h(x)$ in $R[x]$ such that $x^{3}-x^{2}+p x=g(x) \cdot h(x)$ with $\bar{g}(x)=x^{2}$ and $\bar{h}(x)=x-1$ in $(R / \operatorname{rad} R)[x]$. This contradicts the choice of $p$ and completes the proof.

Theorem 1 states that if $R$ is a connected inertial coefficient ring then $R / \operatorname{rad}(R)$ is connected, and so $R$ trivially has the idempotent lifting property. We next use the Pierce representation of $R$ to extend Theorem 1 to an arbitrary inertial coefficient ring.

The decomposition space $X(R)$ of $R$ can be viewed as the quotient space 
obtained from the set of prime ideals of $R$ endowed with the Zariski topology by identifying the points in each connected component. It can be shown [11, Proposition II.12, p. 30] that the open, closed subsets of $X(R)$ form a basis for the topology on $X(R)$, and that any open, closed subset of $X(R)$ is of the form $N(e)=\{x \in X(R): x \subseteq V(R(1-e))\}$, where $e$ is an idempotent of $R$ and $V(R(1-e))$ is the set of prime ideals of $R$ which contain the ideal $R(1-e)$.

Pierce represents $R$ as the ring of global sections of a sheaf over $X(R)$ where at each point $x \in X(R)$ the stalk $R_{x}=R / I(x)$ is a homomorphic image of $R$ and is a connected ring. If $M$ is an $R$-module, $M_{x}=M \otimes_{R} R_{x} \cong$ $M / I(x) M$ is an $R_{x}$-module; if $m \in M, m_{x}$ denotes the image of $m$ in $M_{x}$.

Proposition 2. If $\left(A_{x}, N_{x}\right)$ is an L. I. pair for all $x \in X(R)$, then $(A, N)$ is an L. I. pair.

Proof. Let $u \in A$ be such that $u^{2}-u \in N$. We shall produce an idempotent $e \in N$ such that $u-e \in N$.

Since $R_{x}$ is a flat $R$-module, without ambiguity we can let $N_{x}$ denote the image of $N$ under the canonical homomorphism $A \rightarrow A / I(x) A=A_{x}$. Now $u_{x}=u+I(x) A$ is an element of $A_{x}$ such that $u_{x}+N_{x}$ is an idempotent element of $A_{x} / N_{x}$. Since $\left(A_{x}, N_{x}\right)$ is an L. I. pair and since an idempotent in $A_{x}$ can be lifted to an idempotent in $A$ [11, Proposition II.20, p. 34], there exists an idempotent $f(x) \in A$ such that $u_{x}=[f(x)]_{x}+[n(x)]_{x}$ for some $n(x) \in N$. By [11, Proposition II.16, p. 32] for each $x \in X(R)$ there exists an idempotent $e(x) \in R$ such that $u e(x)=f(x) e(x)+n(x) e(x)$ and $u_{y}=$ $[f(x)]_{y}+[h(x)]_{y}$ for all $y \in N(e(x))$.

By the "partition property" of $X(R)$, the open cover $\{N(e(x))\}$ of $X(R)$ has a finite refinement of disjoint, open, closed sets $\left\{N\left(e_{i}\right)\right\}_{i=1}^{m}$, for some idempotents $e_{i} \in R$, which covers $X(R)$. Since $\left\{N\left(e_{i}\right)\right\}_{i=1}^{m}$ is a cover of $X(R)$ by disjoint sets, it follows that $1=\sum_{i=1}^{m} e_{i}$ and $e_{i} e_{j}=0$ for each $i \neq j$. Furthermore $N\left(e_{i}\right) \subseteq N\left(e\left(x_{i}\right)\right)$ for some $x_{i} \in X(R)$, and thus it can be shown that $e_{i} e\left(x_{i}\right)=e_{i}$; hence, $u e_{i}=f_{i} e_{i}+n_{i} e_{i}$ where $f_{i}=f\left(x_{i}\right)$ and $n_{i}=$ $n\left(x_{i}\right)$. Since the $e_{i}$ are pairwise orthogonal idempotents and each $f_{i}$ is an idempotent, then $e=\sum_{i=1}^{m} f_{i} e_{i}$ is an idempotent. Now

$$
u=\sum_{i=1}^{m} u e_{i}=\sum_{i=1}^{m}\left(f_{i} e_{i}+n_{i} e_{i}\right)=e+\sum_{i=1}^{m} n_{i} e_{i},
$$

and therefore $u-e \in N$.

COROllaRy 3. If $R$ is an inertial coefficient ring then $(R, \operatorname{rad}(R))$ is an L. I. pair.

Proof. Each stalk $R_{x}$ is a connected ring and is an inertial coefficient ring because it is a homomorphic image of $R$. By Theorem $1,\left(R_{x}, \operatorname{rad}\left(R_{x}\right)\right)$ is an L. I. pair for every $x \in X(R)$. Since $(\operatorname{rad} R)_{x} \subseteq \operatorname{rad}\left(R_{x}\right)$ and $R_{x}$ is a commutative ring, then $\left(R_{x},(\operatorname{rad} R)_{x}\right)$ is an L. I. pair for every $x \in X(R)$ [5, 
Corollary 1.3, p. 46]; hence by Proposition 2, $(R, \operatorname{rad}(R))$ is an L. I. pair.

We are now able to prove the general case:

THEOREM 4. Let $R$ be an inertial coefficient ring and $A$ a finitely generated $R$-algebra. Then $(A, N)$ is an L. I. pair.

Proof. By replacing $R / \operatorname{annih}_{R} A$ by $R$ we may assume that $A$ is a faithful $R$-algebra.

Let $u \in A$ be such that $u^{2}-u \in N$. We must show there exists an idempotent $e \in A$ such that $u-e \in N$. Let $B$ denote the $R$-subalgebra of $A$ generated by $u$. Now $B / B \cap N$, being a homomorphic image of $(R / \operatorname{rad} R)[x] /\left\langle x^{2}-x\right\rangle$, is a separable $R$-algebra. Since $B$ is a finitely generated $R$-algebra, $B \cap N \subseteq \operatorname{rad}(B)$ [1, Corollary, p. 126], and hence $B / \operatorname{rad}(B)$ is a separable $R$-algebra. Since $B$ is a commutative $R$-algebra, by [7, see proof of Proposition 3.3, p. 85] $B$ is an inertial coefficient ring, and thus by Corollary $3(B, \operatorname{rad}(B))$ is an L. I. pair. It follows from the commutativity of $B$ that $(B, N \cap B)$ is an L. I. pair. Then $u^{2}-u \in N \cap B$ implies that there exists an idempotent $e \in B$ such that $u-e \in N \cap B$. Then $e \in A$ and $u-e \in N$.

COROllary 5. An inertial coefficient ring is a Hensel ring.

Proof. To show $R$ is a Hensel ring it suffices to show that for any finitely generated, commutative, free $R$-algebra $A,(A, \operatorname{rad}(R) A)$ is an L. I. pair [5, Theorem 4.1, p. 55]. Since $R$ is an inertial coefficient $\operatorname{ring},(A, N)$ is an L. I. pair, and since $\operatorname{rad}(R) A \subseteq N$ and $A$ is commutative, then $(A, \operatorname{rad}(R) A)$ is an L. I. pair.

The following corollary to Theorem 4 follows immediately from [8, Corollary 2, p. 556].

COROllary 6. If $R$ is an inertial coefficient ring and if $S$ and $T$ are two inertial subalgebras of a finitely generated $R$-algebra $A$, then $T=(1+n)^{-1}$. $^{\prime}$ $S(1+n)$ for some $n \in N$.

N. S. Ford has given an example of a ring $R$ and an $R$-algebra $A$ such that $S$ and $T$ are not isomorphic [4].

The following corollary to Theorem 4 follows immediately from [8, Theorem 2, p. 554].

COROLlaRY 7. The following are equivalent properties of a commutative ring $R$ :

(i) For all finitely generated, commutative $R$-algebras $A$ such that $A / N$ is $R$-separable, there exists a separable $R$-subalgebra $S$ of $A$ such that $A=S+$ $N$.

(ii) All finitely generated, commutative $R$-algebras have the idempotent lifting property.

If Ingraham's conjecture is true then it is unnecessary to restrict the 
algebras $A$ in Corollary 7 to commutative $R$-algebras, for then the lifting of idempotents from $A / N$ to $A$ in all finitely generated $R$-algebras $A$ is equivalent to the lifting of the separability of $A / N$ to a separable subalgebra $S$ of $A$ in all finitely generated $R$-algebras.

\section{REFERENCES}

1. G. Azumaya, On maximally central algebras, Nagoya Math. J. 2 (1951), 119-150. MR 12, 669.

2. W. C. Brown and E. C. Ingraham, $A$ characterization of semilocal inertial coefficient rings, Proc. Amer. Math. Soc. 26 (1970), 10-14. MR 41 \# 5354.

3. F. DeMeyer and E. C. Ingraham, Separable algebras over commutative rings, Lecture Notes in Math., vol. 181, Springer-Verlag, Berlin and New York, 1971. MR 43 \#6199.

4. N. S. Ford, Ph. D. Dissertation, Michigan State Univ., July 1972.

5. S. Greco, Algebras over nonlocal Hensel rings, J. Algebra 8 (1968), 45-59. MR 36 \# 1435.

$6 .+$, Algebras over nonlocal Hensel rings. II, J. Algebra 13 (1969), 48-56. MR 39 \#5629.

7. E. C. Ingraham, Inertial subalgebras of algebras over commutative rings, Trans. Amer. Math. Soc. 124 (1966), 77-93. MR 34 \#209.

$8 . \quad$ On the existence and conjugacy of inertial subalgebras, J. Algebra 31 (1974), 547-556. MR 50 \#2245.

9. N. Jacobson, Structure of rings, Amer. Math. Soc. Colloq. Publ., vol. 37, Amer. Math. Soc., Providence, R. I., 1956. MR 18, 373.

10. J.-P. Lafon, Anneaux Henséliens, Bull. Soc. Math. France 91 (1963), 77-107. MR 27 \# 174.

11. A. R. Magid, The separable Galois theory of commutative rings, Dekker, New York, 1974. MR 50 \#563.

12. R. S. Pierce, Modules over commutative regular rings, Mem. Amer. Math. Soc. No. 70, (1967). MR 36 \#151.

Department of Mathematics, Wake Forest University, Winston-Salem, North CAROLINA 27109 